\title{
Avaliação de uma política de estabilização de renda para a agricultura familiar
}

\author{
Rodrigo Vilela Rodrigues* \\ Eduardo Rodrigues de Castro** \\ Erly Cardoso Teixeira***
}

Resumo: A Agricultura brasileira sofreu, durante décadas, forte transferência de renda para outros setores da economia nacional, o que causou empobrecimento do setor agrícola com relação aos demais, fato que foi amenizado pelo crédito rural. No entanto, este era centralizado em grandes produtores, o que proporcionou um hiato entre a renda dos produtores familiares e comerciais. Este trabalho avalia a implementação de uma Política de Estabilização de Renda (PER) para os produtores familiares de mandioca, leite e feijão, com base nos custos e benefícios dessa política, utilizando-se a teoria dos excedentes econômicos. Nesse artigo considera-se a perda dos excedentes dos produtores comerciais que não são beneficiados com a PER. Os resultados demonstraram que atenção diferenciada à agricultura familiar seria boa estratégia para aumentar preços recebidos e a renda dos produtores familiares de mandioca, leite e feijão e que a consideração da perda de excedente dos produtores comerciais e do ganho de excedente dos consumidores reforça o uso dessa política, uma vez que o ganho dos consumidores é maior que a perda de excedente dos produtores comerciais.

\footnotetext{
* D.S.; Professor Adjunto I da Universidade Federal Fluminense - Escola de Engenharia Industrial e Metalúrgica de Volta Redonda, RJ. rvilela@metal.eeimvr.uff.br

** Doutorando em Economia Aplicada - Universidade Federal de Viçosa. edu2110@hotmail.com

*** Ph.D.; Professor Titular da Universidade Federal de Viçosa. teixeira@ufv.br
} 
Palavras-chave: PER, mandioca, leite e feijão, agricultura familiar.

\section{Classificação JEL: Q1, Q18}

Abstract: For decades, the Brazilian agriculture sector has suffered strong income transfers to many of the country's other economic sectors, which caused the impoverishment of many Brazilian farmers. Government sponsored rural credit assistance programs have somewhat redressed this inequity and ameliorated the farmer's plight. However, due to the structure of these credit assistance programs, the majority of their benefits have gone to the country's largest agricultural producers, thereby increasing income disparity found between family and commercial farms. New policies are needed that actually assist the majority of those that work in the Brazilian agriculture sector: the family farmers. This work evaluates effects of implementation of an Income Stabilization Policy (ISP) intended to support family farmers, focusing on cassava, milk, and bean producers. The evaluation is based on the costs and benefits of ISP, with reference to the theory of economic surplus. This article also considers the policy's negative effect on the surplus generated by commercial producers. Study results indicate that implementation of the proposed ISP would increase the prices received for cassava, milk and beans, improve the income of Brazilian family farmers producing these products, and lead to consumer gains that more than offset the losses suffered by large, commercial agriculture producers.

Key words: ISP, cassava, milk and bean, family agriculture.

JEL Classification: Q1, Q18

\section{Introdução}

No contexto de transferência de renda da agricultura para os outros setores da economia, dois fatos podem ser apontados como cruciais: a) o mito de que a flexibilidade de preços e a oferta agrícola tornavam o setor inflacionário; e b) a necessidade de recursos para a industrialização do país. 
Nesse sentido, de 1960 a 1992, foram transferidos, anualmente, $40 \%$ da renda do setor agrícola brasileiro para outros setores, fruto de intervenções diretas e indiretas (LOPES, 1993). Tal fato agravou a situação da agricultura no Brasil, que, como na maioria dos países em desenvolvimento, apresenta sérios problemas de instabilidade de renda no setor.

Essas intervenções explicam pelo menos em parte por que a renda per capita do setor agrícola se situa em apenas um terço da renda do setor não-agrícola (TEIXEIRA, 1994). A política do crédito rural, principal instrumento de compensação utilizado no Brasil nas décadas de 1960 e 1970, foi importante, mas contribuiu pouco para a agricultura familiar, devido à concentração desses créditos nos grandes produtores.

A agricultura familiar é responsável por mais de $40 \%$ da produção agrícola no país, abrigando 35,5\% da População Economicamente Ativa no Agronegócio (FERREIRA et al, 1999). Em 1996, a implantação do PRONAF ocorreu com o objetivo de ampliar a distribuição do crédito e de expandir os investimentos em infra-estrutura para a agricultura familiar.

Considerando-se a significativa participação da agricultura familiar na agricultura nacional e a instabilidade de renda desses produtores, uma Política de Estabilização de Renda (PER) poderia ser uma boa alternativa para a capitalização do setor, acesso a inovações tecnológicas e melhor distribuição de renda no campo. Essa política se baseia na determinação de um preço-meta, calculado como a média móvel dos preços reais recebidos dos últimos 60 meses. A transferência de renda dar-se-ia via governo, que pagaria ao produtor a diferença entre esse preço e o preço de mercado, sempre que o preço-meta fosse maior.

O objetivo deste trabalho é analisar os custos e benefícios da PER, quando aplicada à agricultura familiar, especificamente aos produtores de mandioca, leite e feijão, dada a importância desses produtos na produção agrícola nacional e na mesa do consumidor brasileiro. Nesse caso, entende-se como agricultura familiar o conjunto de todos os produtores com áreas das propriedades menores que 100 hectares, enquanto todos os demais são considerados produtores comerciais.

A PER avaliada neste trabalho auxilia apenas os produtores da agricultura familiar, uma vez que eles receberiam do governo a diferença entre o preço que os consumidores estão dispostos a pagar e o preço- 
meta, enquanto os produtores da agricultura comercial receberiam apenas o preço de mercado.

Com relação a outros trabalhos, como os de FERREIRA et al. (1999), KAM-CHINGS et al. (1995) e ALVES et al. (2002), este apresenta uma novidade, que é a consideração da perda de excedente dos produtores comerciais que não seriam contemplados pela PER, além do ganho dos consumidores que compram seus produtos desse grupo.

Este artigo se divide nesta introdução, seguida pela metodologia; os resultados são apresentados na seqüência e o encerramento se dá nas conclusões.

\section{Metodologia}

O referencial teórico deste trabalho baseia-se nas teorias dos excedentes do consumidor e do produtor, as quais avaliam de que maneira modificações nos preços de bens podem resultar em benefícios ou prejuízos tanto para produtores quanto para consumidores.

De acordo com VARIAN (2000), a teoria do excedente do consumidor é uma abordagem alternativa ao problema da estimativa da utilidade com base na observação do comportamento da demanda. Esse excedente é medido pela diferença entre o preço que o consumidor está disposto a pagar por determinado bem e o preço que ele realmente paga por esse bem.

No caso do excedente do produtor, este seria a diferença entre o preço que o produtor está disposto a vender determinada quantidade de seu produto e o preço pelo qual ele realmente a vende. Esta é também uma abordagem alternativa para medir ganhos de produtores com políticas ou choques (VARIAN, 2000).

Para que seja tomada uma decisão econômica com relação à utilização, ou não, de determinada política, é comum que sejam mensurados os ganhos e as perdas dessa política. O modelo analítico utilizado neste trabalho, com base em WALLACE (1962), compara os ganhos e as perdas de uma política com base nos custos sociais desta.

A política analisada neste estudo assemelha-se ao Plano Brannan, examinado em WALLACE (1962), em que é sugerida a imposição de um preço-meta para determinado produto, acima do preço de equilíbrio de 
mercado desse produto. Nesse caso, os consumidores pagariam um preço condizente com a quantidade demandada, resultante da nova oferta, e a diferença seria paga aos produtores via subsídio governamental.

Com relação ao artigo publicado por KAM-CHINGS et al. (1995), este trabalho apresenta um avanço analítico, visto que são mensuradas as perdas auferidas pelos produtores comerciais não favorecidos pelos subsídios que sustentam o preço-meta, além do ganho dos consumidores desse grupo específico.

Analisando-se, primeiramente, o caso da agricultura familiar (Figura 1), a imposição de um preço-meta (P1) faria com que a produção dos produtores familiares aumentasse para Q1, o que resultaria num custo total da política igual à área P1CDP2. Desse custo, a área P1CBP0 seria transferida à agricultura familiar como ganho de excedente dos produtores, enquanto a área P0BDP2 seria transferida aos consumidores como ganho de excedente, o que resultaria num custo social igual à área $\mathrm{BCD}$, isto é, parte do custo governamental com a PER que não é apropriada pelos produtores ou consumidores.

A Figura 2, por sua vez, apresenta o avanço analítico do trabalho, ou seja, a representação não só da perda de excedente dos produtores comerciais, como também os ganhos de excedente dos consumidores que compram os produtos ofertados pelos produtores comerciais.

Figura 1. Representação gráfica dos custos sociais da PER

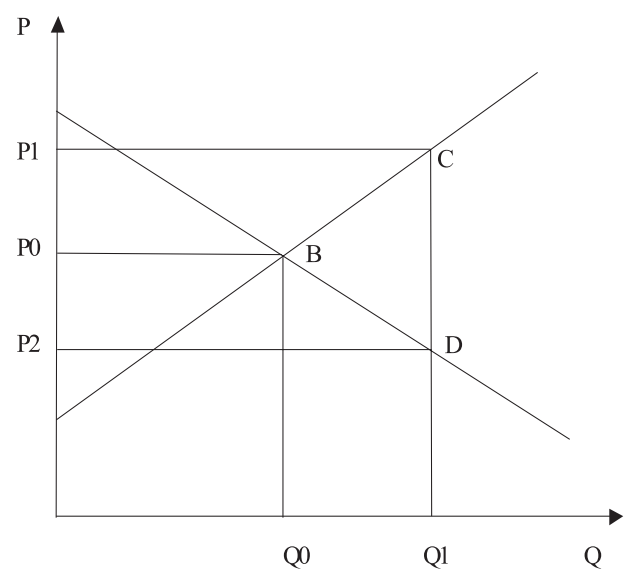


Na Figura 2 (a) a ilustração é adequada aos casos do leite e do feijão, produtos que ao preço de mercado P0 eram importados. Com o pagamento do preço meta (P1), somente aos produtores familiares, o aumento da produção por parte desses reduz as importações. Tal fato derruba o preço interno para $\mathrm{P} 2$, fazendo com que as importações voltem a aumentar. Com a redução do preço de P0 para P2 os produtores comerciais sofreriam uma perda representada, graficamente, pela área P0CDP2. Por outro lado, os consumidores que consomem esses produtos mais baratos teriam um ganho de excedente, representado, na Figura 2, pela área P0ABP2.

Para o caso da mandioca, que apresenta importações e exportações de derivados de mandioca desprezíveis, a Figura 2 (b) é a representação gráfica mais adequada. O pagamento do preço meta (P1) aos produtores familiares reduz o preço de mercado para P2, o que acaba causando uma perda de excedente para os produtores comerciais representada

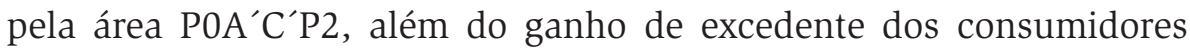

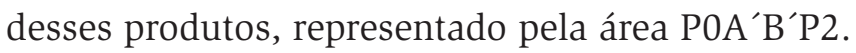

Figura 2. Representação gráfica da perda de excedente dos produtores comerciais e do ganho de excedente dos consumidores desses produtos com importações (a) e sem comércio internacional (b)

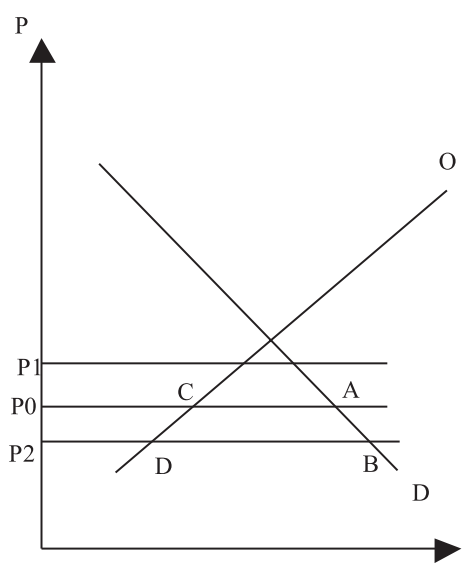

(a)

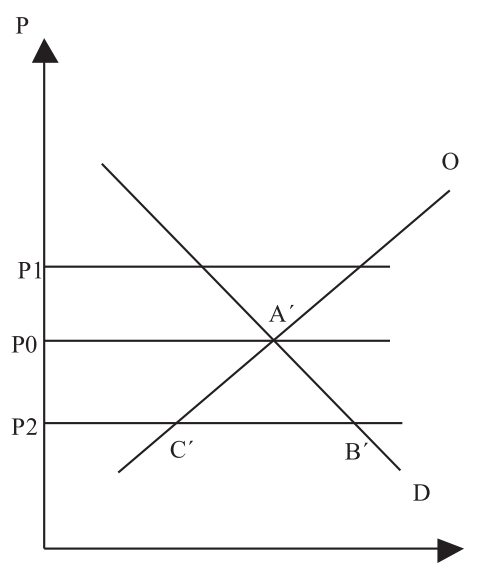

(b)

A fórmula que aproxima o valor do triângulo BCD (Figura 1), condizente com os custos sociais de uma política de estabilização de renda pode ser escrita da seguinte forma: 
$C S=1 / 2 P_{0} Q_{0} r^{2} \varepsilon(1+\varepsilon / \eta)$

em que

$\mathrm{CS}=$ custo social da política;

$\mathrm{P}_{0} \mathrm{Q}_{0}=$ valor da produção sob equilíbrio competitivo;

$\mathrm{r}=$ porcentagem de aumento no preço recebido pelos produtores familiares como preço meta, $r=P_{1}-P_{0} / P_{0}$

$\varepsilon=$ elasticidade-preço da oferta do bem;

$\eta=$ elasticidade-preço da demanda do bem.

A derivação dessa expressão, além da expressão do ganho percentual de renda dos produtores que participam do programa está detalhada no Anexo.

Além dos custos sociais, serão medidos também os custos totais da política, com base na expressão:

$C T=Q_{1} P_{0} r\left(1+\frac{\varepsilon}{\eta}\right)$,

em que

$\mathrm{CT}=$ custos totais da política de subsídios;

$\mathrm{Q}_{1}=$ quantidade ofertada ao preço-meta;

$\mathrm{P}_{0}=$ preço de equilíbrio competitivo.

Outra medida diz respeito ao ganho de excedente dos produtores que seriam beneficiados pela política:

$$
B P=P_{0} Q_{0} r\left(1+\frac{\varepsilon r}{2}\right),
$$

em que

$\boldsymbol{B}=$ benefício ou ganho de excedente dos produtores familiares contemplados pela política;

$\mathrm{P}_{0} \mathrm{Q}_{0}=$ valor da produção auferido com o preço de equilíbrio competitivo.

O ganho de receita por parte dos produtores, quando da adoção da política de preços-meta, poderia ser medido da seguinte maneira: 


$$
K=\varepsilon r^{2}+(\varepsilon+1) r
$$

em que

$\mathrm{K}=$ ganho percentual de receita dos produtores que participassem do programa.

As demais informações importantes podem ser obtidas por diferença entre áreas:

$$
\begin{aligned}
& \text { BT }=\text { CT-CS } \\
& \text { em que } \\
& \text { BT }=\text { benefícios totais }
\end{aligned}
$$

Partindo-se dos benefícios totais, o ganho de excedente do consumidor é dado pela expressão:

$$
\begin{aligned}
& \mathrm{BC}=\mathrm{BT}-\mathrm{BP}, \\
& \text { em que }
\end{aligned}
$$

$\mathrm{BC}=$ benefício ou ganho de excedente dos consumidores (aqueles que consomem os produtos da agricultura familiar) com a queda de preços provocada pela política de preço-meta.

Os trabalhos de FERREIRA et al. (1999), KAM-CHINGS et al. (1995) e ALVES et al. (2002) não levaram em consideração a perda de excedente dos produtores comerciais que não participaram da política, nem os ganhos dos consumidores (que consomem os produtos da agricultura comercial), o que dá uma idéia distorcida dos resultados. Neste trabalho, calcula-se a perda de excedente do produtor comercial devido à redução do preço de mercado resultante da implantação da Política de Estabilização de Renda para a agricultura familiar e o ganho dos consumidores devido à queda no preço pago.

Para tal, deve-se implementar o seguinte procedimento. Primeiramente é necessário o estabelecimento do preço que os consumidores estão dispostos a pagar pelo produto. Tal preço poderia ser obtido por:

$$
P_{2}=P_{0}-\Delta P^{\prime},
$$


em que

$\mathrm{P}_{2}=$ preço que os consumidores estariam dispostos a pagar pelo bem (Figuras 1, 2 (a) e 2 (b)).

$\Delta P^{\prime}=$ queda no preço em decorrência do aumento da produção familiar

Com tal informação, pode-se obter o valor da perda que ocorreria com os produtores que não foram selecionados para participar do programa (o que aumenta custos sociais), uma vez que estes reduziriam sua oferta e receberiam um preço menor pelo seu produto, além do ganho dos consumidores que consomem seus produtos da agricultura comercial (o que reduziria custos sociais) (Figura 2).

Essas novas informações serão somadas aos custos sociais para que um resultado mais realista seja auferido. A diferença entre essas duas novas informações nos dará um adicional ou redutor dos custos sociais, dependendo da relação estabelecida entre o que os produtores comerciais perdem e o que ganham os consumidores da agricultura comercial. Esses novos custos sociais serão comparados aos anteriores, para que se verifique se a variação causada nesses modifica a avaliação da política.

Os preços dos produtos leite, feijão e mandioca foram retirados do IPEA (2003), da mesma fonte veio o Índice Geral de Preços - Deflator Implícito (IGP-DI), cujo período-base, considerado no trabalho, foi de dezembro de 2002.

As séries de quantidades produzidas de todos os produtos foram obtidas do AGRIANUAL (1997) e do AGRIANUAL (2001). As quantidades demandadas são diferentes das ofertadas para feijão e leite; para o feijão, o consumo se encontra no próprio AGRIANUAL, enquanto que para o leite, as importações, em equivalentes litros foram obtidas no site da Associação Brasileira de Produtores de Leite. Quanto à participação de produtores com áreas menores que 100 hectares - agricultura familiar -, as informações vieram do Censo Agropecuário 95/96 (FIBGE, 1996), sendo os demais considerados produtores comerciais. As elasticidades utilizadas nos cálculos dos excedentes dos produtores familiares, comerciais e dos consumidores estão na Tabela 1. 
Tabela 1. Estimativas de elasticidades-preço de oferta e demanda de produtos agrícolas selecionados

\begin{tabular}{l|c|c|c}
\hline \multicolumn{1}{c|}{ Autores } & Produto & Elasticidade de oferta & $\begin{array}{c}\text { Elasticidade } \\
\text { de demanda }\end{array}$ \\
\hline GOMES et al. (1998) & Feijão & 0,38 & $-0,34$ \\
ANDRADE et al. (2001) & Leite & & $-0,54$ \\
GOMES (2003) & Leite & 0,18 & $-0,10$ \\
KAM-CHINGS et al. (1995) & Mandioca & 0,09 & \\
\hline
\end{tabular}

Fonte: Gomes et al. (1998), ANDRADE et al. (2001), GOMES (2003), KAM-CHINGS et al. (1995)

\section{Resultados e Discussões}

Os primeiros resultados são um resumo das variações sofridas por variáveis importantes como receita do produtor, preços do consumidor, preços do produtor e quantidade produzida, sendo importante lembrar que esses resultados foram auferidos pela metodologia na qual não se considerou, ainda, a perda por parte dos produtores comerciais não beneficiados com a política, nem os ganhos dos consumidores desse grupo.

Na Tabela 2 estão resumidos os resultados da cultura da mandioca, no período de 1990 a 2000, excetuando-se os anos de 1995 a 1997, uma vez que, nesses anos, os preços de mercado foram superiores ao preçometa. Com base nos resultados, verifica-se que a Política de Estabilização de Renda teria impacto mais significativo na renda dos produtores familiares, com variação média, no período, de 23,33\%. Os preços recebidos pelos produtores familiares também aumentariam consideravelmente $(20,75 \%)$, em média, no período, enquanto os preços pagos pelos consumidores seriam reduzidos em $12,12 \%$ e a quantidade produzida de mandioca aumentaria apenas 1,87\%.

A Tabela 3 apresenta os resultados para a atividade leite. Neste caso, a Política seria bem mais eficaz para aumentar os preços recebidos pelos produtores familiares, a renda destes e a quantidade produzida, mas foi menos significativa para reduzir os preços pagos pelo consumidor. O aumento de renda auferido pelos produtores de leite, no período de 1990 a 2000, seria da ordem de 49,25\%, em média, fruto de um aumento de preços recebidos de $39,25 \%$ e também da produção $(7,07 \%)$. Os 
preços pagos pelos consumidores seriam pouco afetados e sofreriam variação média no período de apenas - 4,66\% .

Tabela 2. Resumo dos resultados para a agricultura familiar da cultura mandioca, de 1990 a 2000

\begin{tabular}{|c|c|c|c|c|}
\hline & \multicolumn{4}{|c|}{ Variações (\%) } \\
\hline ANO & Renda produtor & Preços produtor & $\begin{array}{c}\text { Preços } \\
\text { consumidor }\end{array}$ & $\begin{array}{c}\text { Quantidade } \\
\text { produzida }\end{array}$ \\
\hline 1990 & 72,28 & 63,03 & $-23,99$ & 5,67 \\
\hline 1991 & 31,37 & 28,12 & $-18,79$ & 2,53 \\
\hline 1992 & 7,77 & 7,09 & $-5,96$ & 0,64 \\
\hline 1993 & 5,68 & 5,19 & $-4,45$ & 0,47 \\
\hline 1994 & 31,66 & 28,38 & $-18,91$ & 2,55 \\
\hline 1998 & 2,78 & 2,55 & $-2,24$ & 0,23 \\
\hline 1999 & 6,26 & 5,72 & $-4,88$ & 0,51 \\
\hline 2000 & 28,82 & 25,89 & $-17,78$ & 2,33 \\
\hline Média & 23,33 & 20,75 & $-12,12$ & 1,87 \\
\hline
\end{tabular}

Fonte: Resultados da pesquisa

OBS: Os anos de 1995, 96 e 97 foram retirados porque o preço de mercado foi maior que o preço-meta

Tabela 3. Resumo dos resultados para a agricultura familiar do leite, de 1990 a 2000

\begin{tabular}{|c|c|c|c|c|}
\hline & \multicolumn{4}{|c|}{ Variações (\%) } \\
\hline ANO & Renda produtor & Preços produtor & $\begin{array}{c}\text { Preços } \\
\text { consumidor }\end{array}$ & $\begin{array}{c}\text { Quantidade } \\
\text { produzida }\end{array}$ \\
\hline 1990 & 62,45 & 49,23 & $-3,89$ & 8,86 \\
\hline 1991 & 60,55 & 47,82 & $-4,12$ & 8,61 \\
\hline 1992 & 66,66 & 52,32 & $-3,30$ & 9,42 \\
\hline 1993 & 35,60 & 28,90 & $-5,32$ & 5,20 \\
\hline 1994 & 37,92 & 30,70 & $-5,36$ & 5,53 \\
\hline 1995 & 31,59 & 25,76 & $-5,16$ & 4,64 \\
\hline 1996 & 46,77 & 37,49 & $-5,23$ & 6,75 \\
\hline 1997 & 62,66 & 49,38 & $-3,86$ & 8,89 \\
\hline 1998 & 55,67 & 44,20 & $-4,64$ & 7,96 \\
\hline 1999 & 48,50 & 38,80 & $-5,15$ & 6,98 \\
\hline 2000 & 33,43 & 27,20 & $-5,24$ & 4,90 \\
\hline Média & 49,25 & 39,25 & $-4,66$ & 7,07 \\
\hline
\end{tabular}

Fonte: Resultados da pesquisa

Os anos de 1994 e 1998 não participam das análises no caso do feijão, uma vez que o preço de mercado superou o preço-meta nesses anos (Tabela 4). 
Tabela 4. Resumo dos resultados para a agricultura familiar da cultura do feijão, de 1990 a 2000

\begin{tabular}{|c|c|c|c|c|}
\hline & \multicolumn{4}{|c|}{ Variações (\%) } \\
\hline ANO & Renda produtor & Preços produtor & $\begin{array}{c}\text { Preços } \\
\text { consumidor }\end{array}$ & $\begin{array}{c}\text { Quantidade } \\
\text { produzida }\end{array}$ \\
\hline 1990 & 83,68 & 52,93 & $-9,66$ & 20,11 \\
\hline 1991 & 91,83 & 57,45 & $-8,92$ & 21,83 \\
\hline 1992 & 1,44 & 1,04 & $-0,51$ & 0,40 \\
\hline 1993 & 25,72 & 17,77 & $-6,99$ & 6,75 \\
\hline 1995 & 102,39 & 63,20 & $-7,63$ & 24,02 \\
\hline 1996 & 56,87 & 37,36 & $-10,31$ & 14,20 \\
\hline 1997 & 78,45 & 49,97 & $-10,00$ & 18,99 \\
\hline 1999 & 45,71 & 30,55 & $-9,68$ & 11,61 \\
\hline 2000 & 115,09 & 69,93 & $-5,62$ & 26,57 \\
\hline Média & 66,80 & 42,25 & $-7,70$ & 16,05 \\
\hline
\end{tabular}

Fonte: Resultados da pesquisa

OBS: os anos de 1994 e 1998 foram retirados porque o preço de mercado foi maior que o preço-meta

Para os produtores de feijão, as variações seriam ainda mais significativas que nos dois casos anteriores. Dada a vigência da política, a renda dos produtores familiares de feijão aumentaria, em média, de $66,80 \%$, com aumento de preços recebidos de $42,25 \%$ e de produção de 16,05\%. Quanto aos preços pagos pelos consumidores, a cultura do feijão apresentaria posição intermediária com relação aos outros dois produtos analisados, com variação negativa de 7,70\% .

A Tabela 5 apresenta os resultados dos benefícios dos produtores da agricultura familiar (contemplados com a política) e dos consumidores, para os três produtos selecionados, no período de 1990 a 2000. Com base nessa tabela, visualiza-se que os maiores benefícios para produtores da agricultura familiar seriam alcançados com a implantação dessa política para o produto leite, cujos benefícios anuais médios, para esses produtores, seriam de R $1.876,42$ milhões, enquanto os consumidores de leite, por sua vez, alcançariam benefícios médios de $\mathrm{R} \$ 215,16$ milhões.

O segundo produto a apresentar os maiores valores para os benefícios de seus produtores familiares foi o feijão, com $\mathrm{R} \$ 1.015,67 \mathrm{mi}-$ lhões, enquanto seus consumidores seriam beneficiados em R \$ 820,14 milhões. Os produtores familiares de mandioca seriam os menos beneficiados com a implementação de uma PER por parte do governo 
federal com $\mathrm{R}$ \$ 509,07 milhões, enquanto seus consumidores seriam mais beneficiados que os consumidores de leite, mas não tanto quanto os consumidores de feijão com R\$294,33 milhões.

Tabela 5. Benefícios dos produtores da agricultura familiar (BPAF) e dos consumidores (BC) com a implementação da Política de Estabilização de Renda, de 1990 a 2000

$\mathrm{R} \$ 1.000,00$

\begin{tabular}{|c|c|c|c|c|c|c|}
\hline & \multicolumn{2}{|c|}{ Mandioca } & \multicolumn{2}{c|}{ Leite } & \multicolumn{2}{c|}{ Feijão } \\
\hline ANO & BPAF & BC & BPAF & BC & BPAF & BC \\
\hline 1990 & $1.535 .884,08$ & $568.501,46$ & $2.571 .266,27$ & $194.511,46$ & $1.346 .048,11$ & $1.079 .468,78$ \\
\hline 1991 & $796.938,68$ & $525.904,71$ & $2.485 .556,07$ & $205.539,62$ & $1.631 .770,04$ & $1.268 .866,13$ \\
\hline 1992 & $199.849,11$ & $167.661,11$ & $2.537 .315,11$ & $152.731,58$ & $38.409,19$ & $42.665,60$ \\
\hline 1993 & $144.643,64$ & $123.708,07$ & $1.487 .333,54$ & $266.775,71$ & $478.638,22$ & $480.938,03$ \\
\hline 1994 & $642.953,11$ & $422.886,04$ & $1.488 .111,65$ & $253.023,86$ & & \\
\hline 1995 & & & $1.293 .142,48$ & $253.081,42$ & $1.563 .239,80$ & $1.168 .113,48$ \\
\hline 1996 & & & $1.881 .414,06$ & $254.101,93$ & $921.556,66$ & $818.914,96$ \\
\hline 1997 & & & $2.207 .106,54$ & $165.218,96$ & $1.099 .999,73$ & $899.841,77$ \\
\hline 1998 & $58.494,42$ & $51.358,83$ & $1.873 .011,45$ & $189.121,74$ & & \\
\hline 1999 & $137.888,12$ & $117.302,44$ & $1.626 .990,81$ & $208.846,26$ & $713.627,04$ & $662.316,16$ \\
\hline 2000 & $555.887,37$ & $377.314,00$ & $1.189 .377,31$ & $223.802,80$ & $1.347 .717,11$ & $960.110,29$ \\
\hline Média & $509.067,32$ & $294.329,58$ & $1.876 .420,48$ & $215.159,58$ & $1.015 .667,32$ & $820.137,24$ \\
\hline
\end{tabular}

Fonte: Resultados da pesquisa

BPAF - Benefícios dos produtores da agricultura familiar

$\mathrm{BC}$ - Benefícios dos consumidores

Com relação aos custos totais da implantação da PER para cada produto, verifica-se que o produto mais caro para o governo seria o leite, com R \$ 2.591,96 milhões anuais em média, enquanto o feijão viria em segundo, com R $\$ 2.343,90$ milhões anuais em média e, por último, a mandioca, com R $\$ 983,43$ milhões anuais em média (Tabela 6).

$\mathrm{O}$ apoio ao feijão é o mais caro à sociedade (maiores custos sociais), com $\mathrm{R} \$ 508,10$ milhões, seguido de perto pelo leite ( $\mathrm{R}$ \$ 500,38 milhões) e pela mandioca, que, por sua vez apresentou custos sociais bem inferiores aos demais produtos (R\$ 180,03 milhões).

A participação dos custos sociais nos custos totais permite verificar se a política é viável ou não, sem a consideração da perda de excedente dos produtores comerciais e do ganho de seus consumidores. Essa variável apresentou resultado mais expressivo para a cultura do feijão $(21,68 \%)$, seguido de perto pelas duas outras atividades, leite $(19,30 \%)$ e mandioca $(18,31 \%)$, demonstrando que, sem considerar-se a perda de 
excedente dos produtores comerciais, os custos que não são absorvidos por produtores familiares ou consumidores representam parcela pequena do custo total.

Tabela 6. Custos totais e custos sociais da Política de Estabilização de Renda para a agricultura familiar das culturas de mandioca, feijão e para o leite, de 1990 a 2000

$\mathrm{R} \$ 1.000,00$

\begin{tabular}{|l|c|c|c|c|c|c|}
\hline & \multicolumn{2}{|c|}{ Mandioca } & \multicolumn{2}{c|}{ Leite } & \multicolumn{2}{c|}{ Feijão } \\
\hline ANO & Custos Sociais & Custos Totais & Custos Sociais & Custos Totais & Custos Sociais & Custos Totais \\
\hline 1990 & $894.279,35$ & $2.998 .664,90$ & $808.021,09$ & $3.573 .798,82$ & $685.383,77$ & $3.110 .900,65$ \\
\hline 1991 & $210.263,85$ & $1.533 .107,24$ & $759.730,58$ & $3.450 .826,27$ & $894.962,66$ & $3.795 .598,83$ \\
\hline 1992 & $13.409,98$ & $380.920,20$ & $845.170,88$ & $3.535 .217,57$ & 423,09 & $81.497,88$ \\
\hline 1993 & $7.111,21$ & $275.462,92$ & $279.270,90$ & $2.033 .380,15$ & $87.113,83$ & $1.046 .690,07$ \\
\hline 1994 & $171.177,76$ & $1.237 .016,91$ & $296.357,75$ & $2.037 .493,27$ & & \\
\hline 1995 & & & $217.031,80$ & $1.763 .255,70$ & $933.930,46$ & $3.665 .283,73$ \\
\hline 1996 & & & $454.922,07$ & $2.590 .438,05$ & $340.419,57$ & $2.080 .891,19$ \\
\hline 1997 & & & $695.711,24$ & $3.068 .036,75$ & $531.564,02$ & $2.531 .405,51$ \\
\hline 1998 & $1.413,35$ & $111.266,60$ & $530.750,50$ & $2.592 .883,69$ & & \\
\hline 1999 & $7.469,08$ & $262.659,64$ & $406.687,82$ & $2.242 .524,88$ & $218.172,58$ & $1.594 .115,78$ \\
\hline 2000 & $135.147,95$ & $1.068 .349,31$ & $210.556,47$ & $1.623 .736,58$ & $880.906,10$ & $3.188 .733,50$ \\
\hline Média & $180.034,07$ & $983.430,96$ & $500.382,83$ & $2.591 .962,88$ & $508.097,34$ & $2.343 .901,90$ \\
\hline
\end{tabular}

Fonte: Resultados da pesquisa

A fim de explorar a análise de custos e benefícios das políticas em termos relativos, implementou-se relação Benefício/Custo, dividindose os resultados dos benefícios dos produtores da agricultura familiar pelos custos sociais de cada política, permitindo, assim, pontuar qual produto apresentou melhor resultado, levando-se em conta também seus custos (Tabela 7).

Com base nos resultados acima, percebe-se que, apesar do leite ter sido o produto em que os produtores da agricultura familiar mais se beneficiaram, a relação desses benefícios com os custos sociais da política foi, apesar de maior que um, a menos significativa (4,20 em média para os anos 90). A política que apresentou melhor relação Benefício/Custo foi aplicada à cultura da mandioca, com média de 13,56 para a década de 1990. A política aplicada ao feijão apresentou relação Benefício/Custo de 12,37 em média nos anos 90 , principalmente por causa do valor de 1992 (90,78), sem o qual seu resultado seria inferior ao do leite. Tais resultados comprovam a relevância de tal análise, uma vez que se 
inverteu completamente a ordem de importância estabelecida pela análise dos benefícios aos produtores da agricultura familiar.

Um dos principais objetivos deste trabalho é acrescentar a esses resultados os efeitos negativos que uma PER teria sobre os produtores comerciais que não participassem da política, fato observado na Tabela 8 .

Tabela 7. Relação Benefício/Custo da Política de Estabilização de Renda para a agricultura familiar das culturas de mandioca, feijão e para o leite, de 1990 a 2000

\begin{tabular}{|c|c|c|c|}
\hline & Mandioca & Leite & Feijão \\
\hline ANO & BPAF/CS & BPAF/CS & BPAF/CS \\
\hline 1990 & 1,72 & 3,18 & 1,96 \\
\hline 1991 & 3,79 & 3,27 & 1,82 \\
\hline 1992 & 14,90 & 3,00 & 90,78 \\
\hline 1993 & 20,34 & 5,33 & 5,49 \\
\hline 1994 & 3,76 & 5,02 & \\
\hline 1995 & & 5,96 & 1,67 \\
\hline 1996 & & 4,14 & 2,71 \\
\hline 1997 & & 3,17 & 2,07 \\
\hline 1998 & 41,39 & 3,53 & \\
\hline 1999 & 18,46 & 4,00 & 3,27 \\
\hline 2000 & 4,11 & 5,65 & 1,53 \\
\hline Média & 13,56 & 4,20 & 12,37 \\
\hline
\end{tabular}

Fonte: Resultados da Pesquisa

BPAF/CS - Relação Benefício/Custo das políticas

Os produtores comerciais de leite seriam os mais prejudicados, com perdas de $\mathrm{R}$ \$ 711,63 milhões, enquanto os de feijão perderiam $\mathrm{R}$ \$ 402,98 milhões e os de mandioca, $\mathrm{R} \$ 137,37$ milhões.

Relacionando-se as perdas dos produtores da agricultura comercial a, respectivamente, benefícios dos produtores da agricultura familiar e benefícios dos consumidores, visualiza-se que a mandioca foi o produto que apresentou menores coeficientes, com 0,30 e 0,43 (Tabela 9). $\mathrm{O}$ leite apresentou a maior relação perdas dos produtores da agricultura comercial/benefícios dos consumidores $(3,50)$, enquanto o feijão teve posição intermediária em ambas relações, com 0,43 para PPAC/BPAF (Perdas dos produtores da agricultura comercial/Benefícios dos produ- 
tores da agricultura familiar) e 0,50 para PPAC/BC (Perdas dos produtores da agricultura comercial/Benefícios dos consumidores).

Tabela 8. Perda de excedente dos produtores de mandioca, leite e feijão da agricultura comercial, com a implantação da PER - 1990 a 2000

\begin{tabular}{|c|c|c|c|}
\hline & & \multicolumn{2}{c|}{ PPAC $\$ 1.000,00$} \\
\hline ANO & Mandioca & Leite & Feijão \\
\hline 1990 & $353.805,95$ & $931.021,37$ & $544.413,28$ \\
\hline 1991 & $227.007,47$ & $907.515,41$ & $638.183,11$ \\
\hline 1992 & $64.144,62$ & $901.953,14$ & $23.304,01$ \\
\hline 1993 & $46.924,04$ & $606.564,58$ & $252.816,05$ \\
\hline 1994 & $182.871,61$ & $600.584,30$ & $585.840,71$ \\
\hline 1995 & & $537.037,92$ & $418.554,32$ \\
\hline 1996 & & $729.907,94$ & $454.748,44$ \\
\hline 1997 & & $798.415,30$ & $260.022,22$ \\
\hline 1998 & $19.260,41$ & $698.670,65$ & $448.958,19$ \\
\hline 1999 & $44.599,47$ & $626.393,68$ & $402.982,26$ \\
\hline 2000 & $160.392,32$ & $489.828,32$ & \\
\hline Média & $137.375,74$ & $711.626,60$ & \\
\hline
\end{tabular}

Fonte: Resultados da pesquisa

PPAC - Perda dos produtores da agricultura comercial

Como se pode verificar na Tabela 10, os ganhos dos consumidores de produtos da agricultura comercial seguiram a mesma configuração das perdas dos produtores comerciais, sendo os consumidores de leite os mais favorecidos com $\mathrm{R} \$ 1.158,96$ milhões, seguidos pelos consumidores de feijão com $\mathrm{R} \$$ 600,30 milhões. Os consumidores de mandioca foram os menos favorecidos com R \$ 142,37 milhões. Comparando-se esses mesmos valores às médias das perdas de excedente dos produtores comerciais, se verifica considerável vantagem em prol dos ganhos dos consumidores com relação à perda dos produtores.

Considerando-se as perdas dos produtores comerciais e os ganhos de seus consumidores, os custos sociais da PER, reduzidos da diferença entre essas informações, para leite e feijão, sofreram quedas consideráveis, fato que não se repetiu para mandioca. O resultado em favor dos consumidores, pelo menos no caso da mandioca pode ser explicado pelo fato da elasticidade-preço da demanda ser maior que a mesma grandeza relativa à oferta, nos casos do leite e do feijão, a diferença 
deveu-se à maior quantidade demandada com relação à ofertada, diferença suprida com importações.

Tabela 9. Relações Perdas dos produtores da agricultura comercial/ Benefícios dos produtores da agricultura familiar e Perdas dos produtores da agricultura comercial/Benefícios dos consumidores para Políticas de Estabilização de Renda para a agricultura familiar das culturas de mandioca, feijão e para o leite, de 1990 a 2000

\begin{tabular}{|c|r|r|r|r|r|r|}
\hline & \multicolumn{2}{|c|}{ Mandioca } & \multicolumn{2}{c|}{ Leite } & \multicolumn{2}{c|}{ Feijão } \\
\hline ANO & PPAC/BPAF & PPAC/BC & PPAC/BPAF & PPAC/BC & PPAC/BPAF & PPAC/BC \\
\hline 1990 & 0,23 & 0,62 & 0,36 & 4,79 & 0,40 & 0,50 \\
\hline 1991 & 0,28 & 0,43 & 0,37 & 4,42 & 0,39 & 0,50 \\
\hline 1992 & 0,32 & 0,38 & 0,36 & 5,91 & 0,61 & 0,55 \\
\hline 1993 & 0,32 & 0,38 & 0,41 & 2,27 & 0,53 & 0,53 \\
\hline 1994 & 0,28 & 0,43 & 0,40 & 2,37 & & \\
\hline 1995 & & & 0,42 & 2,12 & 0,37 & 0,50 \\
\hline 1996 & & & 0,39 & 2,87 & 0,45 & 0,51 \\
\hline 1997 & & & 0,36 & 4,83 & 0,41 & 0,51 \\
\hline 1998 & 0,33 & 0,38 & 0,37 & 3,69 & & \\
\hline 1999 & 0,32 & 0,38 & 0,38 & 3,00 & 0,36 & 0,39 \\
\hline 2000 & 0,29 & 0,43 & 0,41 & 2,19 & 0,33 & 0,47 \\
\hline Média & 0,30 & 0,43 & 0,38 & 3,50 & 0,43 & 0,50 \\
\hline
\end{tabular}

Fonte: Resultados da pesquisa

PPAC/BPAF - relação Perdas dos produtores da agricultura familiar/benefícios dos produtores da agricultura familiar

PPAC/BPAF - relação Perdas dos produtores da agricultura familiar/benefícios dos consumidores

De acordo com a Tabela 11, os custos sociais da mandioca sofreram redução média de 4,59\%, enquanto que para as atividades de leite e feijão, as reduções foram semelhantes entre si e bem maiores, com valores de $109,67 \%$ e 79,62\% respectivamente. Na Tabela 11, por exemplo, o valor de $\mathrm{R} \$ 875,90$ milhões representa os custos sociais finais, em 1990, para o produto mandioca, ou seja, o valor considerando-se as perdas dos produtores comerciais e os ganhos dos consumidores desses produtos. Essa colocação é interessante para que se verifique que, em alguns casos, a redução sobre os custos sociais supera $100 \%$ do seu valor, anulando os custos sociais da política, como no caso do feijão nos anos de 1993 e 1999. 
Tabela 10. Ganho de excedente dos consumidores de mandioca, leite e feijão, que compram seus produtos da agricultura comercial - 1990 a 2000

$\mathrm{R} \$ 1.000,00$

\begin{tabular}{|c|c|c|c|}
\hline & \multicolumn{3}{|c|}{ GCAC } \\
\hline ANO & Mandioca & Leite & Feijão \\
\hline 1990 & $372.188,40$ & $1.382 .659,18$ & $1.024 .664,62$ \\
\hline 1991 & $235.037,01$ & $1.471 .395,28$ & $793.798,03$ \\
\hline 1992 & $64.891,29$ & $1.113 .833,29$ & $23.433,67$ \\
\hline 1993 & $47.334,01$ & $800.500,28$ & $557.466,93$ \\
\hline 1994 & $189.378,68$ & $922.983,35$ & \\
\hline 1995 & & $1.152 .641,28$ & $753.836,85$ \\
\hline 1996 & & $1.350 .726,89$ & $587.929,26$ \\
\hline 1997 & & $1.370 .280,87$ & $525.600,88$ \\
\hline 1998 & $19.345,96$ & $1.262 .581,31$ & \\
\hline 1999 & $45.025,91$ & $1.140 .776,43$ & $605.811,21$ \\
\hline 2000 & $165.761,50$ & $780.165,48$ & $530.152,04$ \\
\hline Média & $142.370,34$ & $1.158 .958,51$ & $600.299,28$ \\
\hline
\end{tabular}

Fonte: Resultados da pesquisa

GCAC - Ganho dos consumidores da agricultura comercial

Tabela 11. Variações nos custos sociais ao se considerarem as perdas de excedente dos produtores comerciais de mandioca, leite e feijão - 1990 a 2000

$\mathrm{R} \$ 1.000,00$

\begin{tabular}{|c|c|c|c|c|c|c|}
\hline & \multicolumn{2}{|c|}{ Mandioca } & \multicolumn{2}{c|}{ Leite } & \multicolumn{2}{c|}{ Feijão } \\
\hline ANO & Custos Sociais & Variação \% & Custos Sociais & Variação \% & Custos Sociais & Variação \% \\
\hline 1990 & $875.896,55$ & $-2,06$ & $356.383,18$ & $-55,89$ & $205.131 .664,28$ & $-70,07$ \\
\hline 1991 & $202.233,47$ & $-3,82$ & $195.850,14$ & $-74,22$ & $739.347 .076,88$ & $-17,39$ \\
\hline 1992 & $12.662,34$ & $-5,57$ & $633.289,85$ & $-25,07$ & $293.339,43$ & $-30,65$ \\
\hline 1993 & $6.701,03$ & $-5,77$ & $85.334,29$ & $-69,44$ & $-217.537 .882,25$ & $-349,72$ \\
\hline 1994 & $164.669,93$ & $-3,80$ & $-26.042,05$ & $-108,79$ & & \\
\hline 1995 & & & $-398.572,35$ & $-283,65$ & $765.933 .862,10$ & $-17,99$ \\
\hline 1996 & & & $-165.896,95$ & $-136,47$ & $171.044 .067,67$ & $-49,75$ \\
\hline 1997 & & & $123.845,43$ & $-82,20$ & $460.711 .559,40$ & $-13,33$ \\
\hline 1998 & $1.327,45$ & $-6,05$ & $-33.160,66$ & $-106,25$ & & \\
\hline 1999 & $7.042,56$ & $-5,71$ & $-107.695,75$ & $-126,48$ & $-127.616 .990,50$ & $-158,49$ \\
\hline 2000 & $129.777,82$ & $-3,97$ & $-79.781,16$ & $-137,89$ & $799.712 .153,01$ & $-9,22$ \\
\hline Média & $175.038,89$ & $-4,59$ & $53.050,36$ & $-109,67$ & $310.779 .872,22$ & $-79,62$ \\
\hline
\end{tabular}

Fonte: Resultados da pesquisa

Os resultados auferidos com essa inovação analítica não inviabilizam a PER em nenhum dos casos, fato que poderia ter ocorrido. Os ganhos dos consumidores sempre excederam as perdas dos produtores 
comerciais, contribuindo para reduzir os custos sociais da política aplicada à agricultura familiar.

\section{Conclusões}

As receitas dos produtores familiares dos três produtos aumentaram, no mínimo, em torno de $20 \%$, com destaque para leite $(49,25 \%)$ e feijão $(66,80 \%)$. Os preços recebidos pelo produtor familiar também tiveram aumento significativo, entre 20,75\% (mandioca) e 42,25\% (feijão); enquanto que nos preços pagos pelos consumidores o produto que apresentou maior queda de preço foi a mandioca $(12,12 \%)$. As variações na oferta seguem as elasticidades-preço, ou seja, a oferta cresceria mais para feijão $(16,05 \%)$, seguido de leite $(7,07 \%)$ e mandioca $(1,87 \%)$.

Quando as variáveis analisadas são os benefícios da PER, tanto para produtores familiares quanto para consumidores há uma configuração bem distinta entre esses dois resultados. No caso dos benefícios para os produtores familiares, os produtores de leite estariam em melhor situação, seguidos pelos produtores de feijão e mandioca. Os consumidores mais beneficiados seriam os de feijão, mandioca e leite, respectivamente.

Os custos sociais da PER, por sua vez, tiveram comportamento semelhante ao dos benefícios dos produtores familiares, com valores próximos entre feijão e leite (de maior custo) e valor bem inferior a esses dois para a mandioca, o que fez com que a mesma fosse a de melhor relação Benefício/Custo, seguida por feijão e leite. As variações dos custos sociais, em decorrência da consideração dos benefícios dos consumidores da agricultura comercial, apresentaram configuração bem distinta entre os produtos selecionados, com valores para mandioca $(-4,59 \%)$, feijão $(-79,62 \%)$, e maior valor para o leite $(-109,67 \%)$. É importante citar que as variações mais significativas para leite e feijão se devem ao montante importado desses produtos, fato que não se aplica à mandioca.

Com base na avaliação desses resultados, pode-se observar que atenção diferenciada à agricultura familiar seria uma boa estratégia para fazer com que os produtores de mandioca, leite e feijão percebam um acréscimo considerável em suas principais variáveis de decisão - preço e renda. Um acréscimo nessas variáveis, uma vez que a agricultura familiar é responsável por $55 \%$ da produção de leite, $71 \%$ da produção de 
feijão e $85 \%$ da produção de mandioca, traria uma série de benefícios não só para a agricultura familiar, mas para o setor agrícola, como capitalização, acesso a novas tecnologias, melhor distribuição de renda na economia como um todo e dentro da agricultura principalmente.

Por outro lado, a consideração das perdas de excedente dos produtores não contemplados com a PER e dos ganhos de excedente dos consumidores de seus produtos fez com que os custos sociais dessas políticas sofressem variação negativa, o que acabou por tornar a implementação dessas políticas ainda mais atraente.

Uma das limitações do trabalho está relacionada com o fato de se considerar que o mercado agrícola funcione como uma concorrência perfeita. Qualquer desvio dessa pressuposição pode fazer com que os resultados sofram mudanças importantes. Os principais problemas a serem considerados nessa questão são a existência de monopólio nos processadores de alimentos, a velocidade de ajuste da oferta à demanda e a incerteza de preços de oferta.

Este trabalho abre espaço para que a mesma análise seja implementada para outros produtos, considerando-se a significância da agricultura familiar no Brasil e o fato de esta apresentar resultados de perda de excedentes de produtores não subsidiados pela PER e de ganhos de consumidores de produtos advindos da agricultura comercial.

\section{Referências Bibliográficas}

ALVES, L.B. GOMES, M.F.M. TEIXEIRA, E.C. LIMA, J.E. Avaliação de um Programa de Garantia de Renda e Crédito Rural na Agricultura Familiar. Revista de Economia e Sociologia Rural, Brasília, vol 40, nº 1 , 29-52, 2002.

ANDRADE, W. S. P., LIMA, J. E., NOGUEIRA, M.A. - Demanda de leite fluído no Brasil no período pós Plano Real. In XXXIX Congresso Brasileiro de Economia e Sociologia Rural. Recife - Pernambuco - 2001.

FERREIRA, A.V. FIGUEIREDO, A.M.R. TEIXEIRA, E.C. Custos e Benefícios de um Programa de Garantia de Renda aplicado ao PRONAF. Revista de Economia e Sociologia Rural, Brasília, vol 37, nº 2, 31-50, 1999. 
FUNDAÇÃO INSTITUTO BRASILEIRO DE GEOGRAFIA E ESTATÍSTICA - FIBGE. Censo Agropecuário 1995-1996. Rio de Janeiro, 1996.

FNP CONSULTORIA E COMÉRCIO - Agrianual 1997. São Paulo: 1998. $435 p$.

FNP CONSULTORIA E COMÉRCIO - Agrianual 2001. São Paulo: 2002. $545 \mathrm{p}$.

GOMES, M. F. M., FERNANDES, L.L., TEIXEIRA, E. C., A política de preços mínimos: custos e benefícios sociais, decisão de plantio e definição de garantia. In: AGUIAR, D. R. D., PINHO, J. B. (Eds). O agronegócio brasileiro: desafios e perspectivas. Brasília: Sociedade Brasileira de Economia e Sociologia Rural - SOBER, 1998 - p. 75 - 88. vol 2.

GOMES, A. Determinantes do preço do leite recebido pelo produtor: uma abordagem de curto e longo prazo. Viçosa: UFV, 2003. Dissertação de Mestrado - Universidade Federal de Viçosa, 2003.

IPEA - Instituto de Pesquisa Econômica Aplicada. [01 mar. 2003]. (http:// www.ipeadata.gov.br).

KAM-CHINGS, M.H.F.L. TEIXEIRA, E.C. Política de Garantia de Renda e Equivalência em Produto: Análise Comparativa de Custos e Benefícios. Revista de Economia e Sociologia Rural, Brasília, vol 33, nº1, 23-36, 1995.

LOPES, M.R. O Poder das Coalizões Políticas de Grupos de Interesse de Bloquear o Desenvolvimento Agrícola. Desenvolvimento Agrícola na Década de 1990 e no Século XXI, Editor: Erly Cardoso Teixeira. FINEP - FAPEMIG - UFV. Viçosa - MG, Impressa Universitária, 1993 p: 105-129.

TEIXEIRA, E.C. Política Agrícola e combate à fome. Conjuntura Econômica. Set. 1994. pg 41-43.

VARIAN, H.R. Microeconomia: Princípios Básicos. $3^{\text {a }}$ ed. Rio de Janeiro: Campus, 2000. 756p.

WALLACE, T.O. Measure of Social costs of agricultural programs. Journal of Farm Economics. Vol 44:p. 580-594, may 1962. 


\section{Anexo}

$C S=1 / 2 P_{0} Q_{0} r^{2} \varepsilon(1+\varepsilon / \eta)$ é a expressão dos custos sociais da PER, que pode ser derivada como segue:

$$
C S=1 / 2 \Delta Q\left(\Delta P+\Delta P^{\prime}\right), \text { em que }
$$

$\Delta Q=$ variação na quantidade ofertada, com a vigência da política $\left(\mathrm{Q}_{0}-\mathrm{Q}_{1}\right)$; $\Delta P=$ variação do preço de equilíbrio para o preço meta, que será recebido pelos produtores beneficiados pela política $\left(\mathrm{P}_{0}-\mathrm{P}_{1}\right)$;

$\Delta P^{\prime}=$ variação do preço de equilíbrio para o preço pago pelos consumidores, após a vigência da política $\left(\mathrm{P}_{0}-\mathrm{P}_{2}\right)$.

Uma vez que $\Delta P$ pode ser escrito como:

$\Delta P=P_{0} r$, e a elasticidade-preço da oferta é dada por:

$\varepsilon=\frac{\Delta Q}{\Delta P} \cdot \frac{P_{0}}{Q_{0}}$

a elasticidade-preço da oferta pode ser reescrita como:

$$
\varepsilon=\frac{\Delta Q}{P_{0} r} \cdot \frac{P_{0}}{Q_{0}} \therefore \Delta Q=r \varepsilon Q_{0}
$$

A expressão para $\Delta P^{\prime}$ pode ser alcançada com a manipulação da elasticidade-preço da demanda:

$$
\eta=\frac{\Delta Q}{\Delta P^{\prime}} \cdot \frac{P_{0}}{Q_{0}} \therefore \eta=\frac{\varepsilon r Q_{0} P_{0}}{\Delta P^{\prime} Q_{0}} \therefore \Delta P^{\prime}=\frac{\varepsilon r P_{0}}{\eta}
$$

Definidas as grandezas $\Delta Q, \Delta P$ e $\Delta P^{\prime}$, pode-se reescrever a fórmula referente ao triângulo $\mathrm{BCD}$, como segue:

$$
C S=1 / 2 \varepsilon r Q_{0}\left(P_{0} r+P_{0} r \frac{\varepsilon}{\eta}\right) \therefore C S=1 / 2 \varepsilon r^{2} P_{0} Q_{0}\left(1+\frac{\varepsilon}{\eta}\right)
$$

A outra expressão que será detalhada diz respeito ao ganho percentual de receita dos produtores familiares beneficiados pela política. 


$$
K=\varepsilon r^{2}+(\varepsilon+1) r
$$

em que

$\mathrm{K}=$ ganho percentual de receita dos produtores que participaram do programa.

A expressão de K pode ser reescrita como:

$$
K=\frac{\Delta R_{1}-\Delta R_{0}}{\Delta R_{0}} \therefore K=\frac{P_{1} Q_{1}-P_{0} Q_{0}}{P_{0} Q_{0}}
$$

em que

$\mathrm{R}_{0}=$ receita do produtor familiar antes da implementação da política;

$\mathrm{R}_{1}=$ receita do produtor familiar após a implementação da política;

$\mathrm{P}_{0} \mathrm{Q}_{0}=$ receita do produtor familiar antes da implementação da política;

$\mathrm{P}_{1} \mathrm{Q}_{1}=$ receita do produtor familiar após a implementação da política.

Sendo:

$$
\begin{aligned}
& \Delta P=r P_{0} \therefore r=\frac{\Delta P_{1}}{P_{0}} \therefore r=\frac{P_{1}-P_{0}}{P_{0}} \\
& r P_{0}=P_{1}-P_{0} \therefore P_{1}=r P_{0}+P_{0}
\end{aligned}
$$

Substituindo-se a expressão 9 em 7, tem-se:

$$
K=\frac{\left(r P_{0}+P_{0}\right) Q_{1}-P_{0} Q_{0}}{P_{0} Q_{0}} \therefore K=\frac{r P_{0} Q_{1}+P_{0} Q_{1}-P_{0} Q_{0}}{P_{0} Q_{0}} \therefore K=\frac{P_{0} Q_{1}(r+1)}{P_{0} Q_{0}}-1
$$

A elasticidade-preço da oferta pode ser escrita como:

$$
\varepsilon=\frac{Q_{1}-Q_{0}}{P_{1}-P_{0}} \cdot \frac{P_{0}}{Q_{0}}
$$

Invertendo-se a expressão 8, tem-se: $\frac{1}{r}=\frac{P_{0}}{P_{1}-P_{0}}$.

Substituindo-se 12 em 11, obtém-se :

$$
\varepsilon=\frac{Q_{1}-Q_{0}}{Q_{0}} \cdot \frac{1}{r} \therefore \varepsilon r=\frac{Q_{1}-Q_{0}}{Q_{0}} \because \frac{Q_{1}}{Q_{0}}=\varepsilon r+1
$$


Substituindo-se 13 em 10, finalmente, tem-se a expressão de ganho percentual de renda dos consumidores participantes da PER:

$$
K=\frac{P_{0} Q_{1}(r+1)}{P_{0} Q_{0}}-1 \therefore K=\varepsilon r+1(r+1)-1 \because K=\varepsilon r^{2}+(\varepsilon+1) r .
$$

Recebido em outubro de 2004 e revisto novembro de 2006. 